\title{
EGFR/HER2 inhibitors effectively reduce the malignant potential of MDR breast cancer evoked by P-gp substrates in vitro and in vivo
}

\author{
YITING JIN $^{1}$, WEI ZHANG ${ }^{1}$, HONGYING WANG ${ }^{1}$, ZIJING ZHANG ${ }^{1}$, \\ CHENGYU CHU ${ }^{1}$, XIUPING LIU ${ }^{2,3}$ and QIANG ZOU ${ }^{1}$ \\ ${ }^{1}$ Department of General Surgery, Huashan Hospital, Fudan University; ${ }^{2}$ Department of Pathology, \\ The Fifth People's Hospital of Shanghai, Fudan University, Shanghai 200240; ${ }^{3}$ Department of Pathology, \\ School of Basic Medical Sciences, Fudan University, Shanghai 200032, P.R. China
}

Received August 8,2015; Accepted September 26, 2015

DOI: $10.3892 / o r .2015 .4444$

\begin{abstract}
Multidrug resistance (MDR) induced by chemotherapy in breast cancer frequently leads to tumor invasion, metastasis and poor clinical outcome. We preliminarily found that the epidermal growth factor receptor (EGFR) is involved in enhancing the malignant potential of MDR breast cancer cells, but the mechanism remains unclear. In the present study, we demonstrated in vitro and in vivo that EGFR/HER2 promote the invasive and metastatic abilities of MDR breast cancer. More importantly, a new function of EGFR/HER2 inhibitors was revealed for the first time, which could improve the treatment efficacy of breast cancer by reversing the MDR process rather than by inhibiting tumor growth. Firstly, using quantitative real-time PCR and western blot analysis, we found that overexpression of EGFR/HER2 in MCF7/Adr cells upregulated CD147 and MMP2/9 at both the transcription and protein expression levels, which promoted tumor cell migration, as determined using an in vitro invasion assay. Secondly, the upregulated levels of CD147 and MMP2/9 were decreased when EGFR/HER 2 activity was inhibited, and therefore tumor invasion was also significantly inhibited. These phenomena were also demonstrated in nude mouse assays. Additionally, in MDR breast cancer patients, we found that overexpression of EGFR and P-gp levels led to shorter overall survival (OS) and disease-free survival (DFS) by IHC assays and Kaplan-Meier survival analysis. In conclusion, EGFR/HER2 play a crucial role in enhancing CD147 and MMP expression to establish favorable conditions for invasion/metastasis in MDR breast cancer. The scope of application of EGFR/HER2 inhibitors may be expanded in EGFR/HER2-positive patients. We
\end{abstract}

Correspondence to: Professor Qiang Zou, Department of General Surgery, Huashan Hospital, Fudan University, 12 Middle Wulumuqi Road, Shanghai 200040, P.R. China

E-mail: zouqiangzq@aliyun.com

Key words: EGFR/HER2, inhibitor, breast cancer, multidrug resistance, metastasis suggest that MDR breast cancer patients may benefit from novel therapies targeting EGFR/HER2.

\section{Introduction}

Breast cancer is one of the most common forms of cancer worldwide (1). Chemotherapy occupies an important position in adjuvant therapy; however, during chemotherapy, multidrug resistance (MDR) can occur, resulting in tumor recurrence and metastasis (2). The correlation and the mechanism between MDR and breast cancer metastasis are not well known. Our previous findings demonstrated that epidermal growth factor receptor (EGFR) may play an important role in regulating the metastasis of MDR tumor cells $(3,4)$.

EGFR is an EGF receptor that is involved in cell proliferation and signal transduction, and belongs to the epidermal growth factor receptor (HER) family. This family includes HER1 (erbB1, EGFR), HER2 (erbB2, NEU), HER3 (erbB3) and HER4 (erbB4). EGFR is overexpressed on the membranes of MDR tumor cells (5). There are many reports on the role of EGFR in tumor invasion and metastasis $(3,4,6)$, but few reports on the correlation between EGFR and drug-resistance and malignant abilities of MDR tumor cells. Inhibitors of EGFR and its family members are often used in inhibiting tumor cell proliferation (7). However, no report has evaluated EGFR/HER2 inhibition for the treatment of reversing the drug-resistance of MDR breast cancer.

In the present study, using cell lines, a nude mouse model and patient data, we demonstrated that paclitaxel/epirubicin chemotherapy may promote MDR breast cancer migration and invasion by upregulating CD147, MMP2 and MMP9. Meanwhile, MDR breast cancer possessed higher expression levels of EGFR and HER2. However, when EGFR and HER2 inhibitors were administered, the expression levels of CD147, MMP2 and MMP9, representative of tumor invasion and metastasis abilities, were downregulated. We also evaluated the effect of EGFR/HER2 inhibitors in reversing drug resistance in vivo. The results suggest a critical role of EGFR/HER2 in the promotion of malignant properties in MDR breast cancer. 
Based on the above findings, we hypothesized that tumor invasion and metastasis induced by MDR may be reversed by inhibiting EGFR/HER2 via the following pathway: P-gp - EGFR - CD147 - MMPs. Inhibitors of EGFR and its family members are often used in inhibiting tumor cell proliferation (7). However, no report has evaluated EGFR/HER2 inhibition for the treatment of reversing the drug-resistance of MDR breast cancer.

Anti-EGFR/HER 2 treatment may be a promising therapeutic strategy in MDR breast cancer. The application of EGFR/HER2 inhibitors may be expanded to P-gp-overexpressing MDR breast cancers in addition to EGFR/HER2-overexpressing cases.

\section{Materials and methods}

Chemicals and reagents. P-gp substrates, including paclitaxel (Taxol; Bristol-Myers Squibb, Wallingford, CT, USA) and epirubicin (Pharmorubicin; Pfizer, Inc., New York, NY, USA), and a non-P-gp substrate, epothilone (Ixempra; Bristol-Myers Squibb), were dissolved in either a special diluent (paclitaxel, epothilone) or in sterile distilled water (epirubicin). The substrates were stored frozen as stock solutions and thawed prior to use. The peak plasma concentrations (PPCs) of paclitaxel, epirubicin and epothilone are $3.65 \mu \mathrm{g} / \mathrm{ml}, 0.4 \mu \mathrm{g} / \mathrm{ml}$ and $252 \mathrm{ng} / \mathrm{ml}$, respectively. EGFR/HER2 tyrosine kinase inhibitor (lapatinib, Tykerb) and HER2 monoclonal antibodies (trastuzumab, Herceptin) were purchased from GlaxoSmithKline, (GSK) (Brentford, UK) and Roche (Basel, Switzerland), and their PPCs are $2.43 \mu \mathrm{g} / \mathrm{ml}$ and $216 \mu \mathrm{g} / \mathrm{ml}$, respectively. All other reagents were of analytical grade and obtained from commercial sources.

Cell culture. MCF7/Adr cells were cultured in RPMI-1640 medium (Gibco-BRL, Karlsruhe, Germany), and MCF7 cells were cultured in Dulbecco's modified Eagle's medium (DMEM) (high glucose; Gibco-BRL) supplemented with $0.01 \mathrm{mg} / \mathrm{ml}$ bovine insulin (Sigma, St. Louis, MO, USA). All cell culture media contained $10 \%$ fetal bovine serum (FBS; PAA Laboratories, Linz, Austria), $100 \mathrm{U} / \mathrm{ml}$ penicillin and $100 \mu \mathrm{g} / \mathrm{ml}$ streptomycin. The cells were plated in 6-well plates at a density of $1 \times 10^{6}$ cells/well and further incubated for $24 \mathrm{~h}$ at $37^{\circ} \mathrm{C}$ in a humidified atmosphere containing $5 \% \mathrm{CO}_{2}$. The medium was then removed and replaced with fresh medium containing paclitaxel, epirubicin and epothilone, respectively, with different PPCs $(0.1,1$ and 10 PPC) for another $48 \mathrm{~h}$. When lapatinib and trastuzumab were required, they were added into the incubation at the PPC for $1 \mathrm{~h}$ before addition of the chemotherapy drugs.

Reverse transcription and quantitative real-time polymerase chain reaction. Total cellular RNA was extracted using the TriPure Isolation reagent (Sangon, Shanghai, China). The RNA samples were subjected to reverse transcription (RT) with $2 \mu \mathrm{g}$ of RNA, Oligo (dT) $)_{18}$, dNTP, and reaction buffer supplied with M-MIV reverse transcriptase (Promega, Madison, WI, USA). Real-time PCR reactions were then performed in $20 \mu \mathrm{l}$ of solution with $2 \mu \mathrm{g}$ of cDNA, $1 \mathrm{mM}$ of each forward and reverse primer and $2 \mathrm{X}$ SYBR-Green Mix (Takara, Shuzo, Kyoto, Japan). Changes in the mRNA
Table I. The primers used for the specific amplifications in real-time PCR assays.

\begin{tabular}{lll}
\hline GAPDH & F: & 5'-CATCAAGAAGGTGGTGAAGC-3' \\
& R: & 5'-GGAAATTGTGAGGGAGATGC-3' \\
CD147 & F: & 5'-CGAGATCCAGTGGTGGTTTG-3' \\
& R: & 5'-TCGTAAGTGCCCGTGTCC-3' \\
MMP2 & F: & 5'-GGCCTCTCCTGACATTGACCTT-3' \\
& R: & 5'-GGCCTCGTATACCGCATCAATC-3' \\
MMP9 & F: & 5'-TTTGACAGCGACAAGAAGTGG-3' \\
& R: & 5'-AGGGCGAGGACCATAGAGG-3' \\
EGFR & F: & 5'-CCAAGGCACGAGTAACAAGC-3' \\
& R: & 5'-CCAAATTCCCAAGGACCACC-3' \\
HER2 & F: & 5'-GCAGCTTCATGTCTGTGCC-3 \\
& R: & 5'-ACAGAGACTCAGACCCTGGC-3
\end{tabular}

F, forward; R, reverse.

expression level were calculated following normalization to the glyceraldehyde-3-phosphate dehydrogenase (GAPDH) mRNA level. Relative gene expression was determined by the fluorescence intensity ratio of the target gene to GAPDH. The primers used for the specific amplifications are shown in Table I.

Immunoblotting. The cells were collected and lysed in a modified RIPA buffer supplemented with 1 tablet $/ 50 \mathrm{ml}$ of Complete Protease Inhibitor Cocktail (Roche Molecular Biochemical, Indianapolis, IN, USA). Total cell lysate (50 $\mu \mathrm{g}$ of protein) was resolved using SDS-PAGE and electrophoretically transferred onto PVDF membranes (Millipore, Bedford, MA, USA). The membranes were blocked in 5\% non-fat milk for $1 \mathrm{~h}$ at room temperature and then incubated overnight with the primary antibodies against EGFR (BD Biosciences, San Jose, CA, USA), HER2 and CD147 (both from Santa Cruz Biotechnology, Santa Cruz, CA, USA), MMP2 (Cell Signaling Technology, Inc., Danvers, MA, USA), MMP9 (Abcam, Cambridge, MA, USA) or $\beta$-actin (Sigma) at $4^{\circ} \mathrm{C}$. The membranes were then incubated for $1 \mathrm{~h}$ at $4^{\circ} \mathrm{C}$ with the appropriate HRP-conjugated secondary antibodies (Invitrogen Life Technologies, Carlsbad, CA, USA). Detection of the protein expression levels using ECL (Pierce, Rockford, IL, USA) was performed according to the manufacturer's protocol.

Cell invasion assay. A Boyden dual chamber assay was performed using Transwell chambers with $8-\mu \mathrm{m}$ pore size membranes (BD Biosciences). A total of $5 \times 10^{4}$ cells was suspended in serum-free media and added to the upper chamber, with serum-containing media added as the chemo-attractant in the lower chamber. The Transwell chamber membranes were coated with $40 \mu 1$ of growth factor-reduced Matrigel (BD Biosciences). After $4 \mathrm{~h}$ at $37^{\circ} \mathrm{C}$ with $5 \% \mathrm{CO}_{2}$, the media and cells remaining in the upper chamber were removed using a cotton swab. The insert was fixed in methanol and stained using hematoxylin and eosin. The number of invading cells was counted in five random fields for each group, and the mean number was calculated. 
A
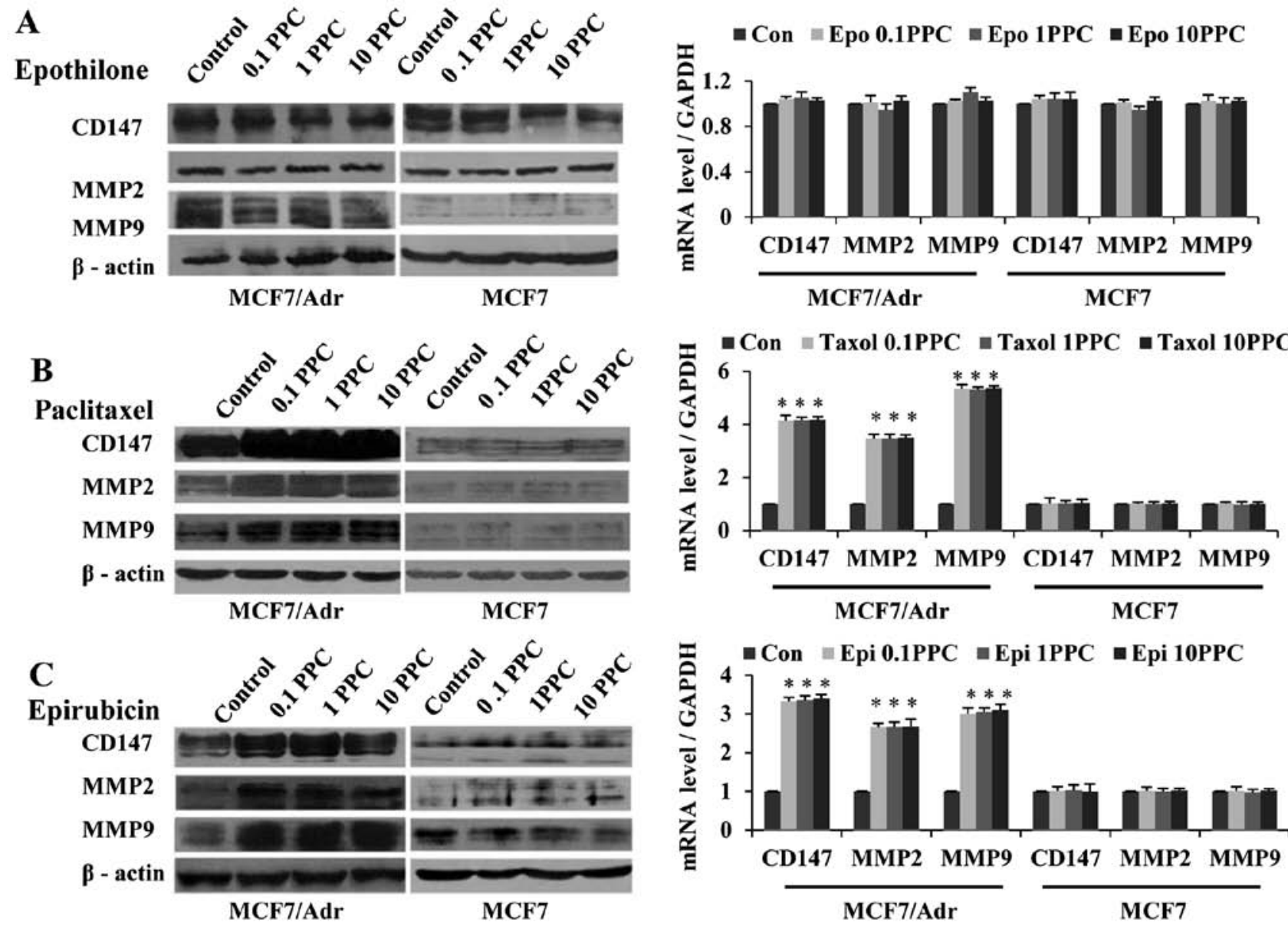

D
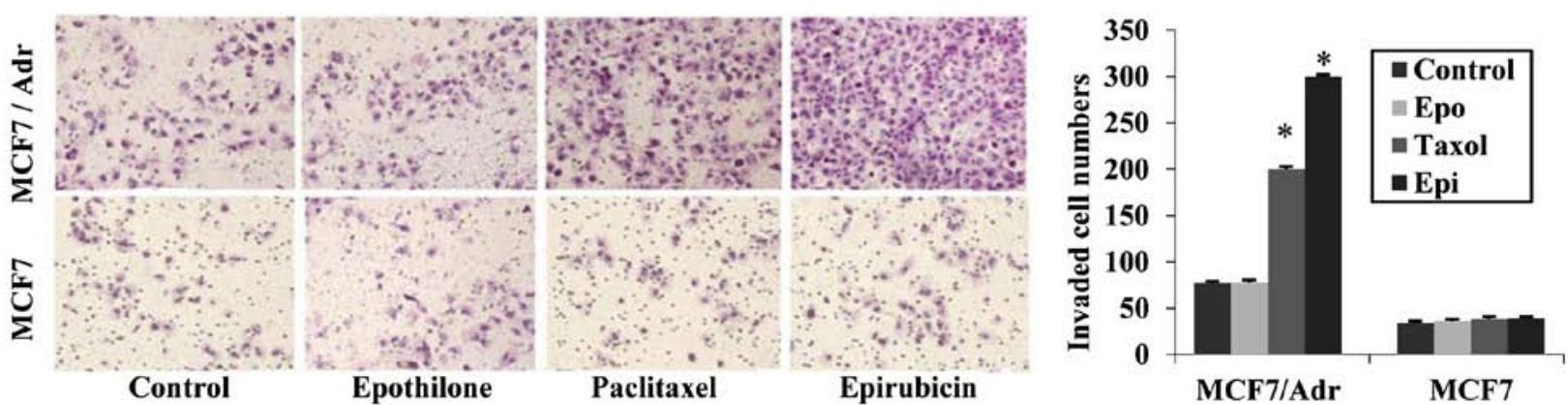

Figure 1. P-gp substrates increase the invasive/metastasis potential of MCF7/Adr cells. (A) Endogenous CD147, MMP2 and MMP9 expression was measured by western blot analysis and real-time PCR in the MCF7/Adr and MCF7 cells exposed to epothilone (0.1, 1 and 10 PPC). (B) CD147, MMP2 and MMP9 expression in the MCF7/Adr and MCF7 cells exposed to paclitaxel (0.1, 1 and 10 PPC). (C) CD147, MMP2 and MMP9 expression in the MCF7/Adr and MCF7 cells exposed to epirubicin (0.1, 1 and 10 PPC). (D) The number of cells that migrated through the permeable Transwell membrane was calculated in five random fields for each group. The bar graphs represent the mean \pm SEM of three independent experiments. (original magnification, $\mathrm{x} 200$ ). The results are representative of three similar experiments. $\mathrm{P}<0.05$ vs. the control group.

Nude mouse model. All animal experiments were performed under the approval of the Animal Experimentation Committee of Fudan University, Shanghai, China. BALB/c female nude mice (age, 4-6 weeks; weight, 18-20 g) were housed under a 12-h light/12-h dark cycle with free access to food and water in sterile conditions. The mice were fed a sterilized murine diet and water. The mice ( $\mathrm{n}=6$ per group) were subcutaneously injected with MCF7/Adr cells $\left(1 \times 10^{7}\right.$ cells/mouse in $200 \mu \mathrm{l}$ of $10 \mathrm{mM}$ phosphate-buffered saline, $\mathrm{pH}$ 7.2) in their second mammary fat pads. When the tumors grew to $1 \mathrm{~cm}^{3}$, the mice were injected with paclitaxel $(18 \mathrm{mg} / \mathrm{kg})$, paclitaxel $(18 \mathrm{mg} / \mathrm{kg})+$ trastuzumab (first time: $36.4 \mathrm{mg} / \mathrm{kg}$, second time: $18.2 \mathrm{mg} / \mathrm{kg}$ ), or $0.9 \%$ normal saline $(0.1 \mathrm{ml})$ as a control, once per week for 4 weeks. The mice were sacrificed during the 5 th week.

Patients and tissue specimens. After approval from our Institutional Review Board, we selected 65 cases of local advanced breast cancer from a cohort of women who had undergone a core-needle biopsy (pathology-confirmed breast invasive ductal carcinoma) and neo-adjuvant chemotherapy (paclitaxel $175 \mathrm{mg} / \mathrm{m}^{2}+$ epirubicin $75 \mathrm{mg} / \mathrm{m}^{2}$, once every 3 weeks for 4 cycles) at our institution between 2009 and 2011 . All patients had received a radical mastectomy after 4 cycles of chemotherapy. According to the Japanese Breast Cancer Society (JBCS) criteria for the histological evaluation of the 

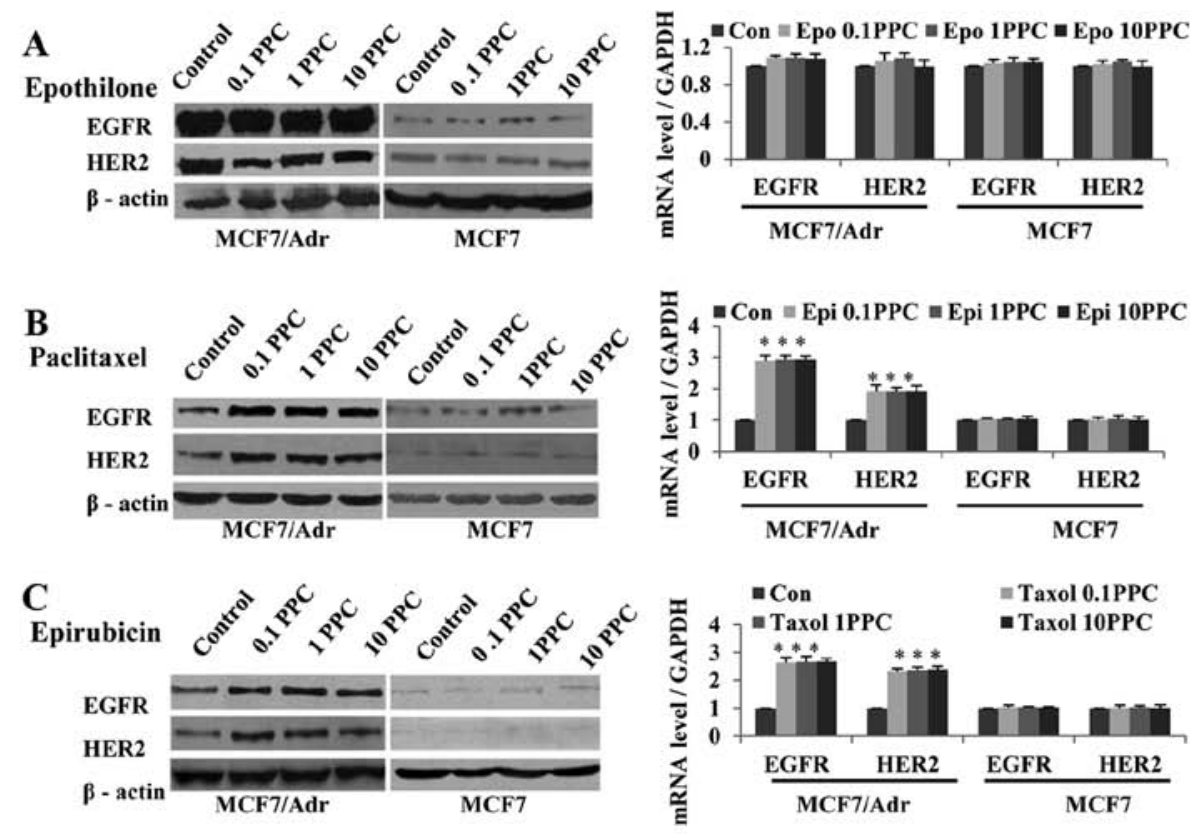

$\mathbf{E}$

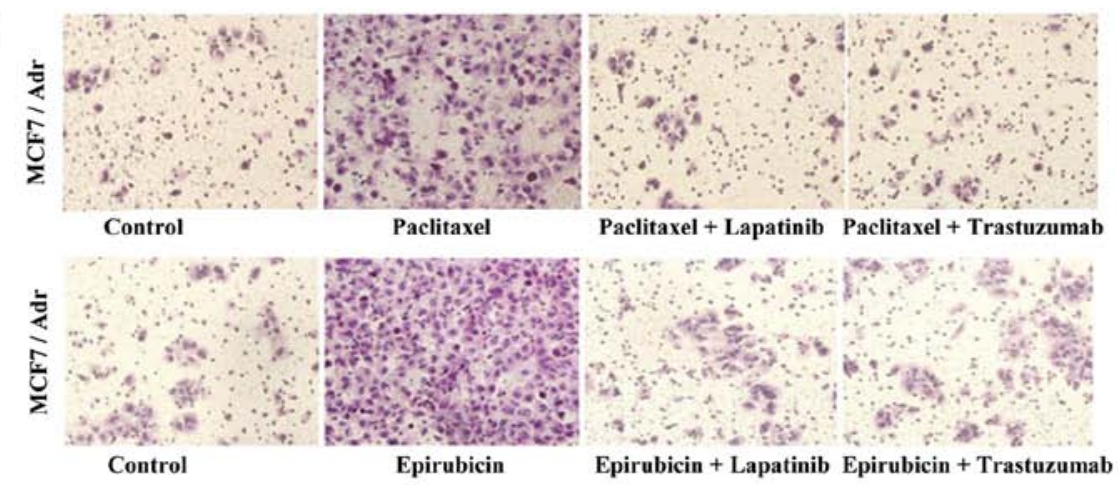

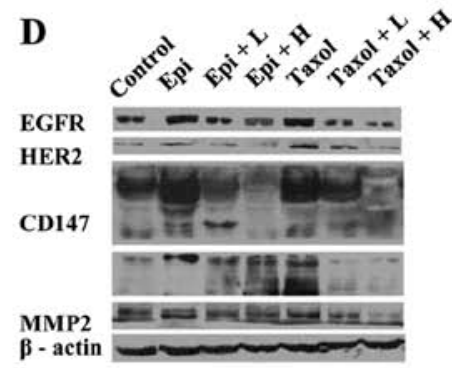
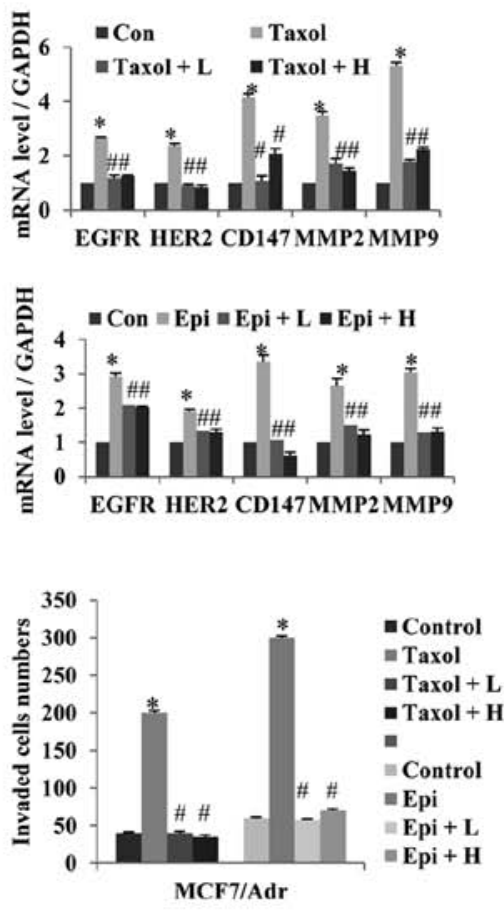

Figure 2. Inhibition of EGFR/HER2 reverses the invasive/metastatic potential of MCF7/Adr cells. (A) Endogenous EGFR and HER2 expression levels were measured by western blot analysis and real-time PCR in the MCF7/Adr and MCF7 cells exposed to epothilone (0.1, 1 and 10 PPC). (B) EGFR and HER2 expression in the MCF7/Adr and MCF7 cells exposed to paclitaxel (0.1, 1 and 10 PPC). (C) EGFR and HER2 expression in the MCF7/Adr and MCF7 cells exposed to epirubicin (0.1, 1 and 10 PPC). (D) MCF7/Adr cells were pre-incubated with lapatinib or trastuzumab for 30 min prior to treatment with the P-gp substrates epirubicin and paclitaxel. The levels of EGFR, HER2, CD147, MMP2, MMP9 were then determined via western blot analysis and real-time PCR. (E) The number of cells that migrated through the permeable membrane was calculated in five random fields for each group. The bar graphs represent the mean \pm SEM of three independent experiments. (original magnification, $\mathrm{x} 200$ ). The results are representative of three similar experiments. ${ }^{*} \mathrm{P}<0.05 \mathrm{vs}$. the control group. ${ }^{\#} \mathrm{P}<0.05$ vs. the epirubicin or paclitaxel group. L, lapatinib; $\mathrm{H}$, trastuzumab.

therapeutic response after neo-adjuvant chemotherapy (8), the cohort was divided into three groups, including pathological complete response (PCR, $n=13)$, partial response $(n=27)$ and no response $(\mathrm{n}=25)$. Tissue specimens before and after chemotherapy were conserved for testing.

Immunohistochemistry (IHC). Paraffin-embedded tissue samples from 18 murine tumors and 65 mammary carcinomas were prepared. The slides were dehydrated in xylene and graded alcohols. Antigen retrieval was performed (EGFR, proteinase $\mathrm{K}$ at room temperature for $20 \mathrm{~min}$; HER2 and P-gp, $0.01 \mathrm{M}$ citrate buffer at $\mathrm{pH} 6.0$ at $95^{\circ} \mathrm{C}$ for $20 \mathrm{~min}$ ). Then, the slides were incubated with diluted primary antibody (anti-EGFR, 1:100; anti-HER2, 1:400; anti-P-gp, 1:50) for $12 \mathrm{~h}$, followed by incubation with a biotinylated secondary antibody for $1 \mathrm{~h}$, peroxidase-labelled streptavidin for $15 \mathrm{~min}$ (LSAB-2 system; Dako, Glostrup, Denmark), and diaminobenzidine and hydrogen peroxide chromogen substrate plus diaminobenzidine enhancer (Dako) for $10 \mathrm{~min}$. The slides were counterstained with Mayer's hematoxylin. The analysis of EGFR, HER2 and P-gp protein expression was performed using an EGFR rabbit polyclonal Ab (BD Biosciences), a HER2 rabbit polyclonal Ab (Santa Cruz Biotechnology) and a P-gp mouse monoclonal Ab (Calbiochem, San Diego, CA, USA). For the statistical analysis, according to the study by Han et al (9), the total staining for EGFR, HER2 and P-gp was scored based on the intensity and percentage of cells with EGFR, HER2 and P-gp cytoplasmic staining using the following scale: score 0 , negative staining for all of the tumor cells; score 1, negative/weak staining for all of the tumor cells or moderate staining in $<10 \%$ of the cells; score 2 , moderate staining in $>30 \%$ but $<70 \%$ of the tumor cells or strong staining within $10 \%$ of the tumor cells; score 3 , moderate staining in $>70 \%$, or strong staining in $>30 \%$ but $<70 \%$ of all the tumor cells; and score 4 , strong staining in $>70 \%$ of all the tumor cells. The reproducibility of EGFR, HER2 and P-gp staining 
A
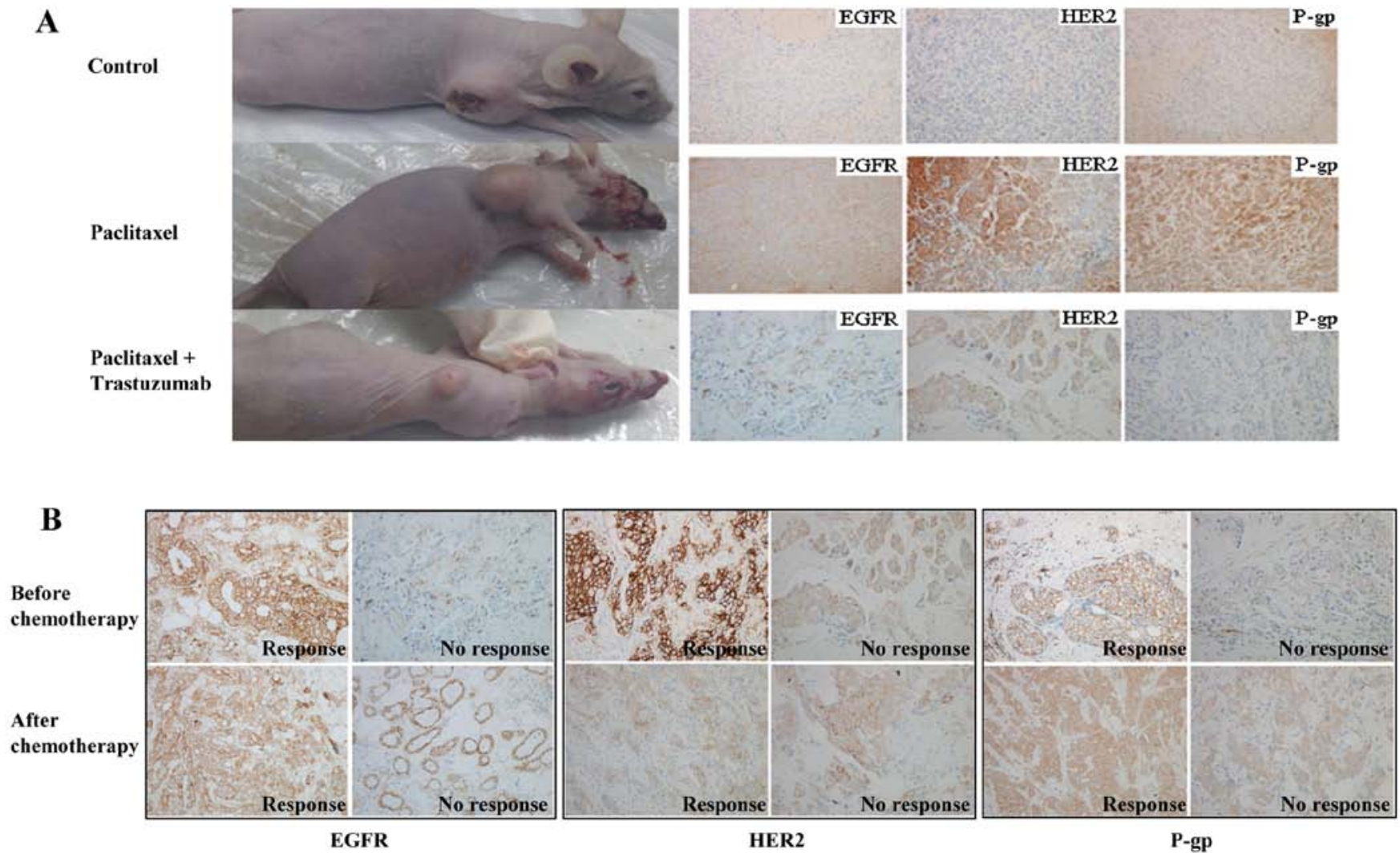

Figure 3. EGFR, HER2 and P-gp expression was measured by immunohistochemistry in nude mice and patient tissues, respectively. (A) Female Balb/c nude mice aged 4-6 weeks were injected in their second mammary fat pads with MCF7/Adr cells. Decreased tumor volumes and weights of the nude mice injected with paclitaxel + trastuzumab compared to the control (0.9\% NS) and paclitaxel group are shown. (B) In human tissues, EGFR, HER2 and P-gp expression levels were measured by IHC before and after chemotherapy. Representative images of H\&E staining of EGFR, HER2 and P-gp of the different groups are shown (original magnification, $\mathrm{x} 200$ ).

was examined between two laboratories (by two independent pathologists).

Statistical analysis. Statistical analysis was carried out using SPSS 19.0 software. All experiments were repeated at least three times, and the results are presented as the mean \pm standard errors (SEM). The differences were analyzed by t-test. Pearson's correlation coefficients were used to determine whether two prognosis-related factors correlated with each other. Kaplan-Meier survival analysis was used to estimate the prognostic relevance. $\mathrm{P}<0.05$ was considered to indicate a statistically significant difference (two-tailed).

\section{Results}

Paclitaxel and epirubicin accelerate the aggressive malignant properties of MCF7/Adr cells by inducing the production of CD147 and MMPs. Following treatment with the non-P-gp substrate epothilone, the production of CD147 and MMP2/9 in both the MCF7/Adr and MCF7 cell lines remained unchanged $(\mathrm{P}>0.05)$ (Fig. 1A). However, incubation with the P-gp substrates (paclitaxel and epirubicin) resulted in a marked increase in CD147 and MMP2/9 at both the transcription and protein level in the MCF7/Adr cells $(\mathrm{P}<0.05)$, while the MCF7 cells did not show a similar response (Fig. 1B and C). Transwell chamber assays showed that MCF7/Adr cells exposed to P-gp substrates displayed a substantially higher invasive ability compared with the corresponding controls $(\mathrm{P}<0.05)$ (Fig. 1D).

EGFR/HER2 inhibitors reverse the enhancement of CD147 and MMPs induced by $P$-gp substrates in vitro. Inhibitors of EGFR and its family members are often used in inhibiting tumor cell proliferation. However, no report has evaluated EGFR/HER2 inhibition for the treatment of reversing the drug-resistance of MDR breast cancer.

To ascertain whether EGFR/HER2 is associated with P-gp substrate-induced CD147 and MMP2/9 upregulation in MDR cells, EGFR and HER2 expression levels were measured in the MCF7/Adr and MCF7 cells after drug treatment. As expected, the results from real-time PCR showed large increases in EGFR and HER2 mRNA only in the MCF7/Adr cells treated with the P-gp substrates. Western blot analysis demonstrated the corresponding alterations at the protein level (Fig. 2A-C), consistent with the enhanced production of CD147 and MMP2/9. There was no similar change in the other groups.

Interestingly, when MCF7/Adr cells were pretreated with lapatinib, an EGFR/HER2 tyrosine kinase inhibitor, or trastuzumab, an HER2 monoclonal antibody, the enhancement of CD147 and MMP2/9 evoked by the P-gp substrates was largely inhibited $(\mathrm{P}<0.05)$, as confirmed using real-time PCR and western blot analysis (Fig. 2D). Additionally, the transcription and protein expression of EGFR and HER2 were also downregulated $(\mathrm{P}<0.05)$ (Fig. 2D). The Transwell chamber assays 
Table II. Statistical quantification and comparison of IHC scores for the expression of EGFR, HER2 and P-gp in nude mice.

\begin{tabular}{lccc}
\hline & \multicolumn{3}{c}{ IHC scores (mean \pm SD) } \\
\cline { 2 - 4 } Group & EFGR & HER2 & P-gp \\
\hline Control & $1.0 \pm 0.89$ & $0.7 \pm 0.52$ & $0.8 \pm 0.75$ \\
Paclitaxel (T) & $2.2 \pm 0.41$ & $1.8 \pm 0.75$ & $2.3 \pm 0.52$ \\
Paclitaxel (T) + Trastuzumab (H) & $1.0 \pm 1.1$ & $1.2 \pm 0.75$ & $1.3 \pm 0.52$ \\
P-values Con vs. T & $0.031^{\mathrm{a}}$ & $0.0098^{\mathrm{a}}$ & $0.0006^{\mathrm{a}}$ \\
& $0.031^{\mathrm{a}}$ & 0.1116 & $0.0119^{\mathrm{a}}$ \\
\hline
\end{tabular}

${ }^{\mathrm{a}} \mathrm{P}<0.05$.

showed that only the MCF7/Adr cells that were pretreated with lapatinib or trastuzumab demonstrated significantly decreased invasive activity $(\mathrm{P}<0.05)($ Fig. $2 \mathrm{E})$.

Inhibition of EGFR and HER2 blocks the upregulation of $P$-gp in the MDR breast cancer in nude mice. The expression levels of EGFR, HER 2 and P-gp were detected using IHC in Balb/c mice bearing MCF7/Adr breast carcinomas (Fig. 3A). Similarly, the upregulation of EGFR, HER2 and P-gp was found only in the group treated with paclitaxel $(\mathrm{P}<0.05)$ (Table II). In vitro, the inhibition of EGFR/HER2 could interrupt the stimulation of CD147, MMP2 and MMP9 evoked by P-gp substrates in the MDR cells. As expected, the expression of EGFR and P-gp was also clearly downregulated in the group co-treated with paclitaxel and trastuzumab $(\mathrm{P}<0.05)$. However, the decrease in HER2 expression was not significant $(\mathrm{P}>0.05)$ (Table II).

EGFR/HER2 are involved in MDR and metastasis of breast cancer in the clinical use of paclitaxel and epirubicin. The treatment of MDR breast cancer with P-gp substrates could adversely affect the function of $\mathrm{P}$-gp and inhibit the degradation of EGFR, which increases the expression of CD147 and MMPs and promotes tumor cell invasion/metastasis. To further validate this hypothesis, 65 local advanced breast cancer (LABC) cases undergoing P-gp substrate chemotherapy (paclitaxel and epirubicin) were chosen for the present study. The expression levels of EGFR, HER2 and P-gp were compared via IHC (Fig. 3B). According to the chemo-response, the patients were divided into 3 groups: pathological complete response (PCR; $n=13)$, partial response $(n=27)$ and no response $(n=25)$. Upregulation of EGFR and P-gp after chemotherapy was only observed in the no response group $(\mathrm{P}<0.05)$ (Table III). Furthermore, we analyzed the relationship between chemo-response and various clinicopathological factors of the 65 cases: estrogen receptor (ER), progestrone receptor (PR) and $\mathrm{Ki67}$. The no response group exhibited increasing lymph node metastasis $(\mathrm{P}<0.01)$ (Table IV). However, in the partial response and PCR groups, there were fewer lymph node metastases. There was no statistical correlation between EGFR and ER, PR and Ki67, and HER2 and P-gp (P>0.05) (Table IV). In the PCR group, due to a lack of tumor tissue after chemotherapy, the differential comparison before and after chemotherapy could not be conducted. The above results revealed that P-gp substrate chemotherapeutic drugs can promote the malignant

Table III. Statistical quantification and comparison of IHC scores of the variation of EGFR, HER2 and P-gp before or after chemotherapy.

\begin{tabular}{|c|c|c|c|c|c|c|c|c|c|}
\hline & \multicolumn{2}{|c|}{ EGFR } & \multirow[b]{2}{*}{ P-value } & \multicolumn{2}{|c|}{ HER2 } & \multirow[b]{2}{*}{ P-value } & \multicolumn{2}{|c|}{ P-gp } & \multirow[b]{2}{*}{ P-value } \\
\hline & Before & After & & Before & After & & Before & After & \\
\hline PCR ( 13 cases $)$ & 0.63 & N/A & N/A & 2.5 & N/A & N/A & 0.5 & N/A & N/A \\
\hline Response (27 cases) & 0.593 & 0.556 & 0.866 & 2.63 & 2.185 & $0.02^{\mathrm{a}}$ & 0.926 & 0.778 & 0.46 \\
\hline No response ( 25 cases) & 0.72 & 1.16 & $0.046^{\mathrm{a}}$ & 2.08 & 2.08 & 1.000 & 1.12 & 1.6 & $0.037^{\mathrm{a}}$ \\
\hline
\end{tabular}

${ }^{\mathrm{a}} \mathrm{P}<0.05$. PCR, pathological complete response; IHC, immunohistochemistry; N/A, not applicable.

Table IV. Statistical quantification and comparison of the expression of ER/PR and Ki67 and the status of LN metastasis after chemotherapy.

\begin{tabular}{|c|c|c|c|c|c|c|}
\hline & \multicolumn{2}{|c|}{ ER/PR (cases) } & \multirow[b]{2}{*}{ Ki67 (mean \pm SD) $\%$} & \multicolumn{3}{|c|}{ LN metastasis (cases) } \\
\hline & + & - & & $(-)$ & $1-3(+)$ & $>3(+)$ \\
\hline PCR (13 cases) & 6 & 7 & $37.1429 \pm 18.898$ & 8 & 4 & 1 \\
\hline Response (27 cases) & 19 & 8 & $26.214 \pm 22.202$ & 13 & 8 & 6 \\
\hline No response ( 25 cases) & 15 & 10 & $35.4 \pm 26.824$ & 3 & 13 & 9 \\
\hline P-value & \multicolumn{2}{|c|}{0.341} & 0.489 & & $0.005^{\mathrm{a}}$ & \\
\hline
\end{tabular}

${ }^{\mathrm{a}} \mathrm{P}<0.05$. PCR, pathological complete response; LN, lymph node; ER, estrogen receptor; PR, progestrone receptor. 
A
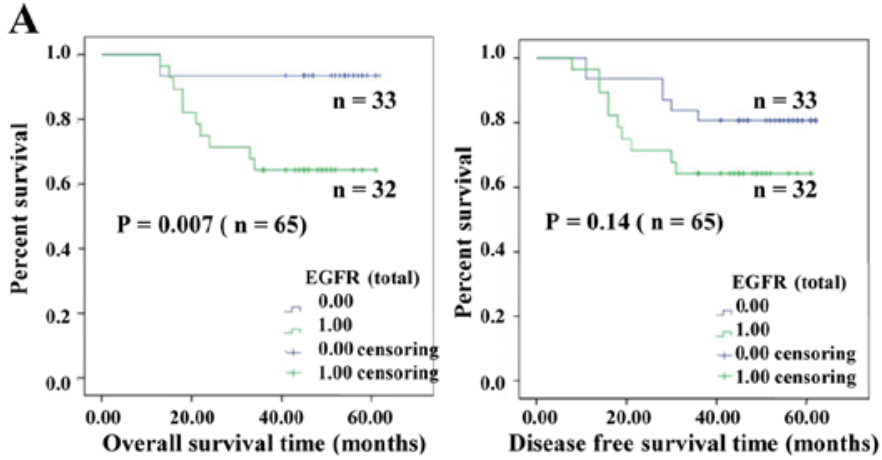

B

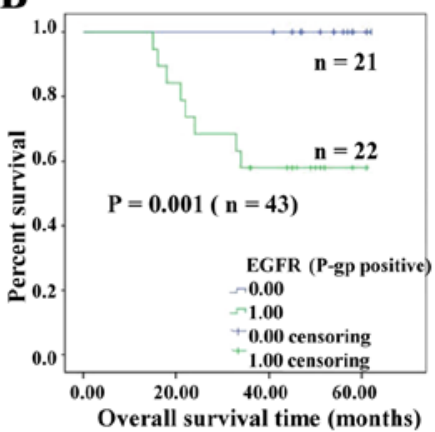

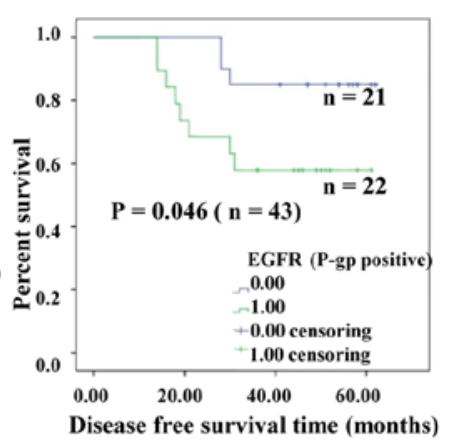

C

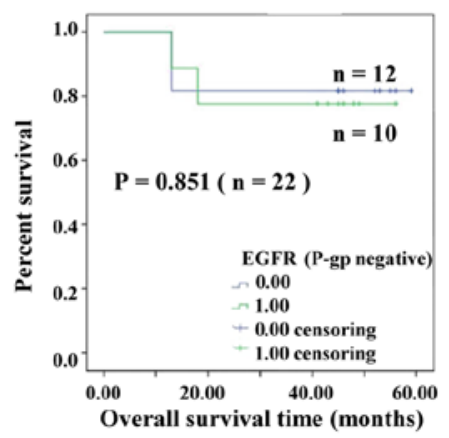

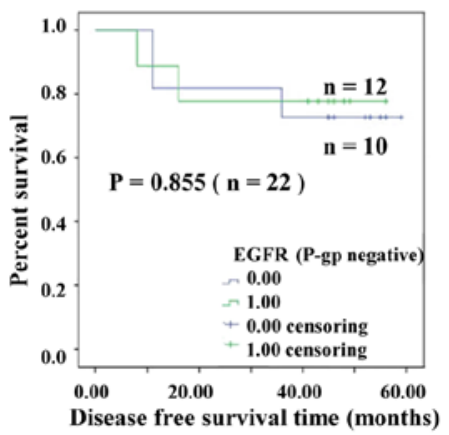

D

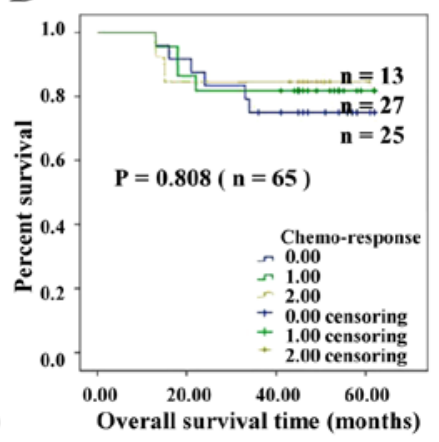

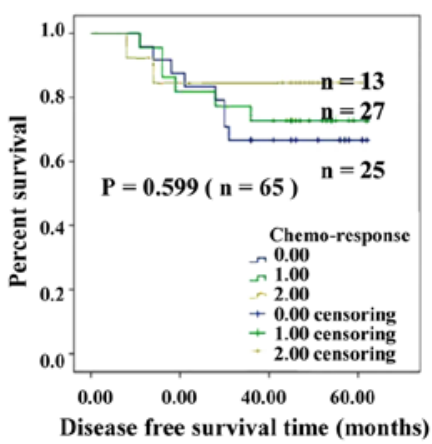

Figure 4. Kaplan-Meier plot of overall survival (OS) and disease-free survival (DFS) times of the 65 breast cancer patients. (A-C) Kaplan-Meier plots of OS and DFS of 65 patients with breast carcinomas stratified by EGFR expression level. The tissues were scored as 0 (negative/weak staining for all of the tumor cells) or 1 (moderate or strong staining of the tumor cells). (A) The total 65 patients. (B) The $43 \mathrm{P}$-gp-positive patients. (C) The $22 \mathrm{P}$-gp-negative patients. (D) Kaplan-Meier plot of OS and DFS of the 65 patients stratified by chemo-response. The tissues were scored as 0 (no response), 1 (partial response) or 2 (PCR). A log-rank test demonstrated a significant difference between the groups.

properties of MDR breast cancer in the clinic, and EGFR and HER2 may be involved in the progression.

Survival analysis reveals that EGFR is a poor prognostic factor of MDR breast cancer patients, especially in P-gppositive tumors. A survival analysis was conducted in 65 cases. Kaplan-Meier plots for OS and DFS stratified by EGFR and P-gp expression levels before chemotherapy are shown in Fig. 4A-C. There was no correlation between HER 2 and OS/DFS (data not shown). In the total cases, we found that higher EGFR expression was associated with shorter OS time $(\mathrm{P}<0.01)$ (Fig. 4A). Furthermore, we separated the patients according to $\mathrm{P}$-gppositive or -negative status. In the P-gp-positive group $(n=43)$, EGFR expression levels were associated with shorter OS $(\mathrm{P}<0.01)$ and shorter DFS times $(\mathrm{P}<0.05)$ (Fig. 4B); however, in the P-gp-negative group $(\mathrm{n}=22)$, there was no correlation between EGFR and OS/DFS times (P>0.05) (Fig. 4C). The results revealed that EGFR is a poor prognostic factor in MDR breast cancer patients, especially in P-gp-positive tumors.

The survival analyses also revealed a trend between poorer chemo-response and shorter OS and DFS, although this finding was not statistically significant (Fig. 4D), most likely due to the small number of cases.

\section{Discussion}

Chemotherapy plays a crucial role in the systemic treatment of breast cancer. However, the American Cancer Society has reported that $>90 \%$ of cancer patients do not fully respond to chemotherapy agents, and MDR during the course of treatment can result in treatment failure (10). Our previous study revealed the correlation between MDR and invasion/metastasis, and MDR breast cancer cells possessed stronger migration/invasion abilities, which could lead to poorer outcomes in clinical treatment $(3,4)$. Therefore, in the clinic, breast cancer chemo-resistance and metastasis are the most urgent problems that call for immediate solutions; however, the mechanisms involved in the enhanced invasion/metastasis abilities of breast cancer following MDR remain unclear.

P-gp overexpression on the MDR tumor cell membrane is one of the main mechanisms of MDR (11). Unfortunately, commonly used drugs for breast cancer chemotherapy, such as doxorubicin, taxane and vincristine, are all P-gp substrates $(12,13)$. Recently, many studies have indicated a connection between drug resistance and tumor invasion/metastasis (14). In our previous study, we also found that MDR cells expressed increased levels of CD147 and MMPs compared with sensitive cells. Moreover, CD147 is not only closely related to invasion/metastasis but is also involved in the regulation of drug resistance induced by P-gp substrates $(3,4)$. We aimed to define the mechanism connecting CD147 and P-gp.

EGFR and HER2 are ErbB family members. A high association between EGFR and HER2 expression and poor prognosis was reported in breast cancer patients treated with different chemotherapeutic strategies but not in a non-chemotherapy group (15), indicating that EGFR expression is likely 
connected to chemotherapy application. We previously reported that EGFR may participate in the regulation of CD147 in MCF7/Adr cells $(6,16)$. Correspondingly, in this study, we found that EGFR and HER2 were expressed at higher levels in drug-resistant MCF7/Adr breast cancer cells than in the sensitive MCF7 cells when treated with P-gp substrates. Additionally, CD147, MMP2 and MMP9 were also expressed at higher levels. Transwell chamber assays showed greater invasion and metastasis of MCF7/Adr cells; however, no changes occurred upon treatment with the non-P-gp substrates or in sensitive cells. Clearly, P-gp substrates may accelerate the aggressive malignant properties of drug-resistant cells MCF7/Adr through inducing CD147 and MMP2/9 production. Thus, EGFR and HER2 are involved in the enhanced invasiveness and metastasis of MDR breast cancer induced by P-gp substrates.

Interestingly, the expression of EGFR, HER2, CD147 and MMP2/9 in the MCF7/Adr cells was apparently reversed when the cells were pretreated with an EGFR/HER2 tyrosine kinase inhibitor (lapatinib) and an HER2 monoclonal antibody (trastuzumab), and the invasive/metastatic abilities of the cells were also significantly decreased. Using a nude mouse model, we also found that the expression levels of EGFR and P-gp were substantially lower following treatment with an EGFR/HER2 inhibitor. In line with our findings, a causal effect of EGFR/HER2 inhibition in MDR breast cancer has been shown to prevent the promotion of MDR and invasion/metastasis induced by P-gp substrates. In conclusion, we hypothesized that the activation of $\mathrm{P}$-gp function decreases the degradation of EGFR/HER2, which leads to the upregulation of CD147. At present, the main purpose of EGFR/HER2 inhibitors is to inhibit tumor cell growth, but our observations suggest a new function. Inhibition of induced invasion and metastasis in MDR tumors is more important than the inhibition of cell growth.

All of the above observations indicate that EGFR/HER2 play an important role in enhancing CD147 and MMP expression to establish favourable conditions for cell migration/invasion not only in generalized breast cancer cells but also in MDR breast cancer cells, indicating that MDR patients with P-gp overexpression may benefit from novel therapies targeting EGFR/HER2.

A clinical experiment was performed to investigate the above hypothesis. We found that the patients who did not respond to neoadjuvant chemotherapy had higher EGFR and P-gp expression levels and an increase in lymph node metastasis, while the response group displayed the opposite results. The survival analyses confirmed that shorter OS and DFS times accompanied increased P-gp and EGFR expression; however, in our trial, there was no difference in HER2 between the different curative effect groups. The present study revealed that MDR breast cancer may produce more P-gp and EGFR, enhance MDR and the invasive/metastatic ability of the tumors via the P-gp - EGFR - CD147 - MMP signaling pathway, thus leading to more invasion and poorer survival.

In summary, inappropriate clinical breast cancer chemotherapy leads not only to unnecessary medical expenses but also an enhanced ability for cancer metastasis. EGFR/HER2 inhibitors reversed the drug-resistance of MDR breast cancer. Therefore, a better understanding of the molecular mecha- nisms of action of EGFR/HER2 were revealed in the present study. These findings may contribute to the development of further strategies for the integration of EGFR/HER2 inhibitors with chemotherapy to potentiate their anticancer activity, particularly in P-gp-positive MDR breast cancer patients.

\section{Acknowledgements}

The present study was supported by grants from the National Nature Science Foundation of China (no. 30872971) and Shanghai Municipal Science and Technology Commission (no. 134119a8502). We thank the members of our laboratory for the helpful discussions.

\section{References}

1. Siegel RL, Miller KD and Jemal A: Cancer statistics, 2015. CA Cancer J Clin 65: 5-29, 2015.

2. Shustik C, Dalton W and Gros P: P-glycoprotein-mediated multidrug resistance in tumor cells: Biochemistry, clinical relevance and modulation. Mol Aspects Med 16: 1-78, 1995.

3. Li QQ, Wang WJ, Xu JD, Cao XX, Chen Q, Yang JM and Xu ZD: Involvement of CD147 in regulation of multidrug resistance to P-gp substrate drugs and in vitro invasion in breast cancer cells. Cancer Sci 98: 1064-1069, 2007.

4. Li QQ, Wang WJ, Xu JD, Cao XX, Chen Q, Yang JM and Xu ZD: Up-regulation of CD147 and matrix metalloproteinase-2, -9 induced by P-glycoprotein substrates in multidrug resistant breast cancer cells. Cancer Sci 98: 1767-1774, 2007.

5. Araújo AP, Catarino R, Ribeiro R, Pereira D, Pinto D and Medeiros R: Epidermal growth factor genetic variation associated with advanced cervical cancer in younger women. Am J Clin Oncol 35: 247-250, 2012.

6. Xu JW, Li QQ, Tao LL, Cheng YY, Yu J, Chen Q, Liu XP and $\mathrm{Xu} \mathrm{ZD}$ : Involvement of EGFR in the promotion of malignant properties in multidrug resistant breast cancer cells. Int $\mathrm{J}$ Oncol 39: 1501-1509, 2011.

7. Gazdar AF: Epidermal growth factor receptor inhibition in lung cancer: The evolving role of individualized therapy. Cancer Metastasis Rev 29: 37-48, 2010.

8. Horii R, Honma N, Ogiya A, Kozuka Y, Fukuda T, Yoshida M, Ohsumi S and Mukai H; Japanese Breast Cancer Society: The Japanese Breast Cancer Society Clinical Practice Guideline for pathological diagnosis of breast cancer. Breast Cancer 22: 59-65, 2015.

9. Han HJ, Russo J, Kohwi Y and Kohwi-Shigematsu T: SATB1 reprogrammes gene expression to promote breast tumour growth and metastasis. Nature 452: 187-193, 2008.

10. DeSantis CE, Lin CC, Mariotto AB, Siegel RL, Stein KD, Kramer JL, Alteri R, Robbins AS and Jemal A: Cancer treatment and survivorship statistics, 2014. CA Cancer J Clin 64: 252-271, 2014.

11. Ambudkar SV, Kimchi-Sarfaty C, Sauna ZE and Gottesman MM: P-glycoprotein: From genomics to mechanism. Oncogene 22: 7468-7485, 2003.

12. Chiou JF, Liang JA, Hsu WH, Wang JJ, Ho ST and Kao A: Comparing the relationship of Taxol-based chemotherapy response with P-glycoprotein and lung resistance-related protein expression in non-small cell lung cancer. Lung 181: 267-273, 2003.

13. Szachowicz-Petelska B, Figaszewski Z and Lewandowski W: Mechanisms of transport across cell membranes of complexes contained in antitumour drugs. Int J Pharm 222: 169-182, 2001.

14. Yang JM, Yang GY, Medina DJ, Vassil AD, Liao J and Hait WN: Treatment of multidrug resistant (MDR1) murine leukemia with P-glycoprotein substrates accelerates the course of the disease. Biochem Biophys Res Commun 266: 167-173, 1999.

15. Rimawi MF, Shetty PB, Weiss HL, Schiff R, Osborne CK, Chamness GC and Elledge RM: Epidermal growth factor receptor expression in breast cancer association with biologic phenotype and clinical outcomes. Cancer 116: 1234-1242, 2010.

16. Menashi S, Serova M, Ma L, Vignot S, Mourah S and Calvo F: Regulation of extracellular matrix metalloproteinase inducer and matrix metalloproteinase expression by amphiregulin in transformed human breast epithelial cells. Cancer Res 63: 7575-7580, 2003. 